\title{
Droplet microfluidics with magnetic beads \\ A new tool to investigate drug-protein interactions
}

\section{Journal Article}

Author(s):

Lombardi, Dario; Dittrich, Petra S. (iD

Publication date:

2010

Permanent link:

https://doi.org/10.3929/ethz-b-000026415

Rights / license:

$\underline{\text { In Copyright - Non-Commercial Use Permitted }}$

Originally published in:

Analytical and Bioanalytical Chemistry 399(1), https://doi.org/10.1007/s00216-010-4302-7 


\title{
Droplet microfluidics with magnetic beads: a new tool to investigate drug-protein interactions
}

\author{
Dario Lombardi • Petra S. Dittrich
}

Received: 16 August 2010 /Revised: 2 October 2010 /Accepted: 5 October 2010 /Published online: 29 October 2010

(C) Springer-Verlag 2010

\begin{abstract}
In this study, we give the proof of concept for a method to determine binding constants of compounds in solution. By implementing a technique based on magnetic beads with a microfluidic device for segmented flow generation, we demonstrate, for individual droplets, fast, robust and complete separation of the magnetic beads. The beads are used as a carrier for one binding partner and hence, any bound molecule is separated likewise, while the segmentation into small microdroplets ensures fast mixing, and opens future prospects for droplet-wise analysis of drug candidate libraries. We employ the method for characterization of drug-protein binding, here warfarin to human serum albumin. The approach lays the basis for a microfluidic droplet-based screening device aimed at investigating the interactions of drugs with specific targets including enzymes and cells. Furthermore, the continuous method could be employed for various applications, such as binding assays, kinetic studies, and single cell analysis, in which rapid removal of a reactive component is required.
\end{abstract}

Keywords Microfluidics · Digital microfluidics · Droplet splitting · Magnetic beads · Serum albumin · Warfarin . Equilibrium constants $\cdot$ Drug-protein interactions

Electronic supplementary material The online version of this article (doi:10.1007/s00216-010-4302-7) contains supplementary material, which is available to authorized users.

D. Lombardi $\cdot$ P. S. Dittrich $(\bowtie)$

Department of Chemistry and Applied Biosciences, ETH Zurich, Wolfgang-Pauli-Str. 10,

8093 Zurich, Switzerland

e-mail: dittrich@org.chem.ethz.ch

\section{Introduction}

Pharmacokinetic studies focus on the fate of a drug after administration to a patient. Various aspects such as the absorption, distribution, metabolism, and excretion (ADME) properties of the drug influence its efficiency. The absorption and distribution of a drug depend significantly on the extent and kinetics of its interactions with specific components of the organism such as proteins and other biomacromolecules or lipid membranes. In particular, serum albumin, one of the most abundant proteins in the blood [1], binds reversibly to an incredible variety of ligands and drugs. In fact, the extensive understanding of the albumin-binding behavior of a particular therapeutic agent is important for its ADME properties [2].

Recently, increasing attention has been paid to apply superparamagnetic iron oxide micro- or nanoparticles (magnetic beads, MBs) for binding assays [3, 4] including the characterization of ligand-serum albumin interactions [5]. The increasing number of commercially available magnetic beads with different surface groups gives further opportunity to extend the applications in the fields of biochemistry and biotechnology [6-8]. However, conventional liquid handling devices suffer from the inability to study fast kinetics, which often restricts the applications of MB-based assays [9].

On the other hand, miniaturized systems based on microfluidic technology designed to carry out experiments in continuous flow have received increasing interest. Traditional bottlenecks in workup and purifications are addressed with the promising prospect to perform all required steps in a single device, thereby reducing sample consumption and reaction/workup time, while reliability and reproducibility is enhanced $[10,11]$.

Microfluidic devices facilitate the handling of small liquid volumes, most impressively shown in the realization of segmented flow, in which homogeneous droplets with 
volumes of $\mathrm{pL}$ to $\mathrm{nL}$ are injected into an immiscible carrier fluid [12-14]. Each droplet, typically an aqueous fluid embedded in a hydrophobic carrier solution, corresponds to an individual microreactor, with enhanced mixing and heat transfer performances compared to large-scale reactors. Various functional modules for microfluidic droplet generation, splitting, fusion, and droplet analysis have been presented in the past and applied for numerous applications such as kinetic investigations, crystallization, and single cell analysis [11, 1518]. These droplet microfluidic approaches have a great potential for continuous screening applications in the drug discovery, development, and testing process [19-22].

The implementation of microfluidic technology with the manipulation of magnetic beads has been shown for numerous applications [23-25]. However, only few studies exist, where magnetic beads were used for concentration or separation of bound analytes. In these examples, droplets were manipulated on a surface by means of electrowetting on dielectrics, which is certainly a powerful technique yet not suitable for continuous, high-throughput droplet manipulation [26, 27].

With the final goal to develop a microfluidic device dedicated to reveal the ADME properties of a drug, we introduce here a simple, continuous and robust method to determine the binding mechanisms and kinetics of a ligand to a protein. The key idea of our device is the introduction of magnetic beads, coated with the target protein, into microdroplets. Such microreactors are completely surrounded by the carrier, which prevents non-specific interactions with the surfaces of the microchip. The magnetic beads and the binding partner are introduced into the droplets and the reaction equilibrium of binding and dissociation events is reached. Finally, the droplets are approaching a T-junction, where they are symmetrically split into two droplets of equal volume. Placing a magnet next to one of the two outlet channels results in selective separation of the beads into one of the daughter droplets (Fig. 1). The affinity (binding) constant can be derived from the concentration ratio of the ligand molecules in the two daughter droplets. In order to demonstrate the functioning of the device, we determine the affinity constant $K_{\mathrm{A}}$ of warfarin, an anticoagulant drug, to human serum albumin (HSA) that is immobilized on the surface of magnetic beads (HSA_MBs). We used radio-labeled ${ }^{14} \mathrm{C}$-warfarin to determine the concentration before and after splitting, and validate the results with the conventional bulk method.

\section{Experimental}

\section{Chip fabrication}

The master form of the microfluidic devices was made from a silicon wafer coated with the negative photoresist SU-

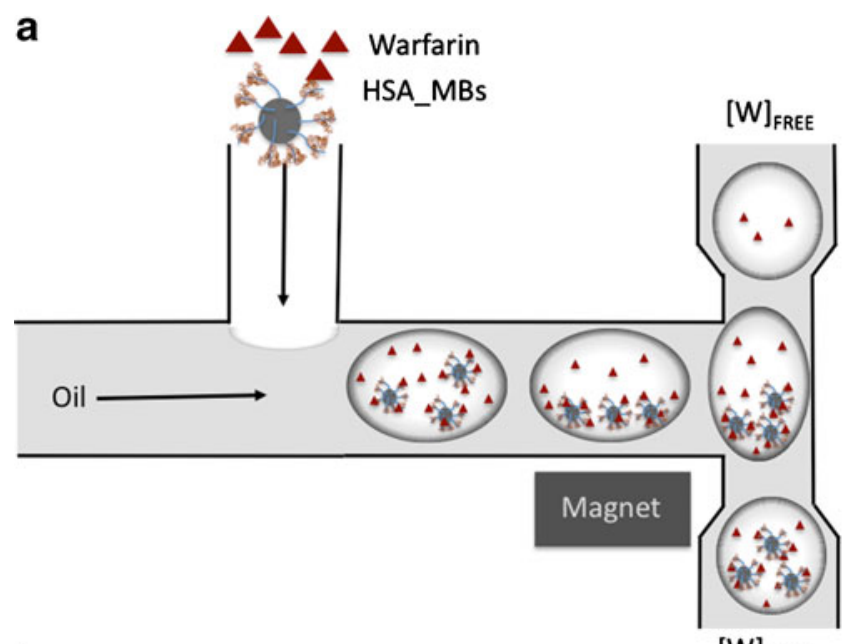

b

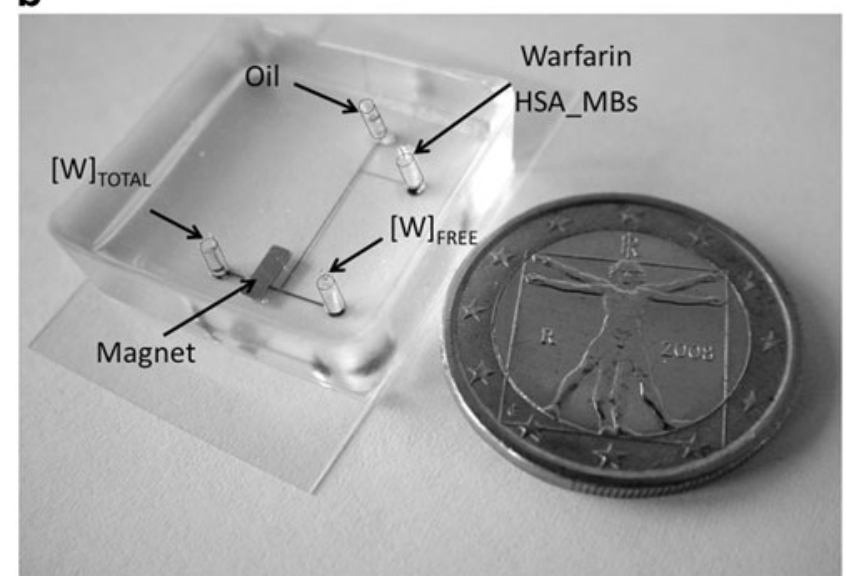

Fig. 1 a Scheme of the method (not to scale). Magnetic beads coated with human serum albumin (HSA_MBs) and the drug (warfarin) are injected into a stream of oil to form microdroplets. Daughter droplets are formed at the T-junction with identical volume, but unequal content, while exposed asymmetrically to a magnetic field. Measuring the free and total (bound+free) warfarin concentrations ([W] $]_{\mathrm{FREE}}$ and $\left.[\mathrm{W}]_{\text {TOTAL }}\right)$ in the outlets finally enables the determination of the association constant. b Photograph of the microdevice (with a one Euro coin (back side) for comparison)

8 that was structured by using standard photolithographic techniques [28]. The final microdevices were made of the elastomer poly(dimethylsiloxane) (PDMS, Sylgard 184, Dow Corning) by molding from the master form. For this, PDMS oligomer and curing agent were mixed at a ratio of 10:1 $(w / w)$, degassed for a few minutes in a desiccator, poured over the master to form a thin layer that was cured at $100{ }^{\circ} \mathrm{C}$ for a few minutes. A neodymium N45 magnet $5 \times$ $1.5 \times 1 \mathrm{~mm}$, nickel coated (Q-05-1.5-01-N, Supermagnete, Switzerland) was positioned on the PDMS microchip (magnetic poles are directing to top and bottom side of the chip) and another portion of degassed PDMS was poured over the thin layer and the magnet to form a second layer of about $0.8 \mathrm{~cm}$. After further curing at $100{ }^{\circ} \mathrm{C}$ for several minutes, the microchip was removed from the 
master form, and $1.5-\mathrm{mm}$ holes were punched for fluidic access. A cleaning process including ethanol ultrasonication, deionized water and nitrogen washing was used to remove PDMS. The channels had a height of $50 \mu \mathrm{m}$ and widths ranging between 50 and $200 \mu \mathrm{m}$. The structured PDMS devices were assembled with glass cover slides $(24 \times 40 \mathrm{~mm}$, Menzel) by bonding after treatment in an oxygen plasma. The microfluidic device was positioned on an inverted microscope (Olympus IX 71) for video analysis by means of a charge-coupled device camera (EMCCD DV-887, Andor).

Mineral oil (4\% $w / w$ Span 80, SIGMA) was chosen as the carrier phase and injected into the device by an external syringe pump (neMESYS, Cetoni, Germany) equipped with $1 \mathrm{~mL}$ plastic syringes, connected via polyethylene tubing to the inlets of the microchip. Alternative carrier oils (e.g., fluorinated oils or silicone oils) could be used depending on the lipophilicity of analytes in order to prevent the partitioning between aqueous and organic phase [29,30].

Preparation of human serum albumin magnetic beads (HSA_MBs)

Amine-terminated MBs (McMag $1 \mu \mathrm{m}$, Bioclone) were used for the immobilization of HSA (A9511, Sigma) according to the previously described method [31]. Briefly, $50 \mathrm{mg}$ of MBs were washed three times with $3 \mathrm{~mL}$ coupling buffer $(10 \mathrm{mM}$ pyridine, $\mathrm{pH} 5.5)$ in a $4 \mathrm{~mL}$ reaction tube. After the washing procedure, the MBs were resuspended in $2 \mathrm{~mL}$ of $5 \%$ glutaraldehyde and gently shaken for $3 \mathrm{~h}$. Next, the supernatant was removed and the MBs were washed three times in $3 \mathrm{~mL}$ of coupling buffer. A solution of $10 \mathrm{mg}$ HSA in $1 \mathrm{~mL}$ of coupling buffer was added to the MBs and gently shaken for $24 \mathrm{~h}$. After the coupling procedure, the supernatant was removed and $4 \mathrm{~mL}$ of the reaction stop buffer ( $1 \mathrm{M}$ glycine, $\mathrm{pH} 8.0$ ) was added and gently rotated for $30 \mathrm{~min}$. The supernatant was removed and the MBs were washed three times and stored in $1 \mathrm{~mL} 10 \mathrm{mM}$ PBS buffer, $\mathrm{pH}$ 7.4. The Sigma protein quantification kit (77371, Sigma) was employed to measure the amount of HSA immobilized on the magnetic beads, which resulted in $24.8 \pm 10.7 \mu \mathrm{g}$ of HSA $/ \mathrm{mg}$ of MBs. Per HSA molecule immobilized on a magnetic bead, we assumed 2.8 binding sites [5].

Preparation of fluorescently labeled magnetic beads

One milligram of streptavidinidated dynabeads, MyOne (Invitrogen) were washed three times and resuspended in $1 \mathrm{~mL}$ PBS buffer. Then $5 \mu \mathrm{l}$ of a stock solution of biotinylated fluorescein (Sigma) in DMSO was added to the beads to a final concentration of $1.5 \mathrm{mM}$. After $30 \mathrm{~min}$ incubation with gentle agitation, the beads were washed three times and resuspended in $1 \mathrm{~mL}$ PBS buffer.

Warfarin binding studies to HSA_MBs in a microdevice

In order to perform the microchip-based determination of the affinity constant we mixed the drug (warfarin) and the ligand (HSA) to allow the chemical equilibrium to be reached before injection into the device. This procedure, instead of the mixing into the droplets, allows control bench-experiments to be performed on the same samples (see below) however, different chip designs are available for on-chip mixing $[19,20]$. Just before starting the experiment, $3 \mathrm{~mL}$ solution of about $100 \mathrm{nM}$ radio-labeled ${ }^{14} \mathrm{C}$-warfarin (CFA449, Amersham) in $10 \mathrm{mM}$ PBS buffer $(\mathrm{pH}$ 7.4) was added to $10 \mathrm{mg}$ of protein-coated beads. Then, the solution was gently shaken for $1 \mathrm{~h}$. Next, $300 \mu \mathrm{L}$ of the working solution were loaded into a $1-\mathrm{mL}$ syringe, connected to the microchip and injected into the carrier phase using a T-junction geometry (Fig. 2a) [32]. Syringe pumps were used to control flow rates; $2 \mu \mathrm{l} / \mathrm{min}$ for the aqueous phase and $1 \mu \mathrm{l} / \mathrm{min}$ for the oil phase typically resulted in $30 \mathrm{drops} / \mathrm{s}$.

After formation, the droplets were split into two small volume copies (Fig. 2b and Movie S1). While the liquid phase was symmetrically split, the MBs were selectively directed to the channel positioned on the side of the neodymium magnet. The volume of the beads was calculated to be $0.2 \%$ of the total volume of the droplet, thus, it was considered negligible. The outlets were connected to tubings thus allowing the emulsions to be collected into two tubes.

Next, the tubes were vigorously shaken in order to resuspend the water and $100 \mu \mathrm{L}$ of each tube aliquot was transferred into scintillation vials (566740, Beckman). Then, $3 \mathrm{~mL}$ of liquid scintillation counting cocktail (Ultima Gold, Packard) were added to each vial. The amount of radio-labeled warfarin in the two samples was measured with a scintillation counter (LS6800, Beckman).

In order to provide control experiments, the concentrations of free and bound warfarin were measured with a bulk method [5]. The tube containing the working solution was vigorously shaken and $100 \mu \mathrm{L}$ of the magnetic beads suspension were transferred into a scintillation vial. Then, the tube was placed into the magnetic separator (Imagnet, BD Biosciences) for $3 \mathrm{~min}$, thus an aliquot of $100 \mu \mathrm{L}$ of the supernatant was transferred into a scintillation vial. The amount of warfarin in the two vials was quantified as described above. Control experiments performed with protein-free MBs revealed a negligible binding of warfarin to the MBs, which is in accordance with previously published results [5]. 
Fig. 2 Microscope images of droplets containing fluorescently labeled magnetic beads. a, b After formation of the droplets, beads are circulating inside the microdroplet. c Once the beads are exposed to a magnetic field, the beads accumulate on one side of the droplet. The image demonstrates how fast this accumulation occurs. d Splitting of droplets into daughter droplets of equal volume at a T-junction. The beads are exclusively withdrawn to one side. Note the clustering of fluorescent beads just before the splitting at the front and the middle section of the droplet, as well as the formation of bead chains after splitting into the direction of the magnetic field, which are typical observations (scale bars $100 \mu \mathrm{m}$ )
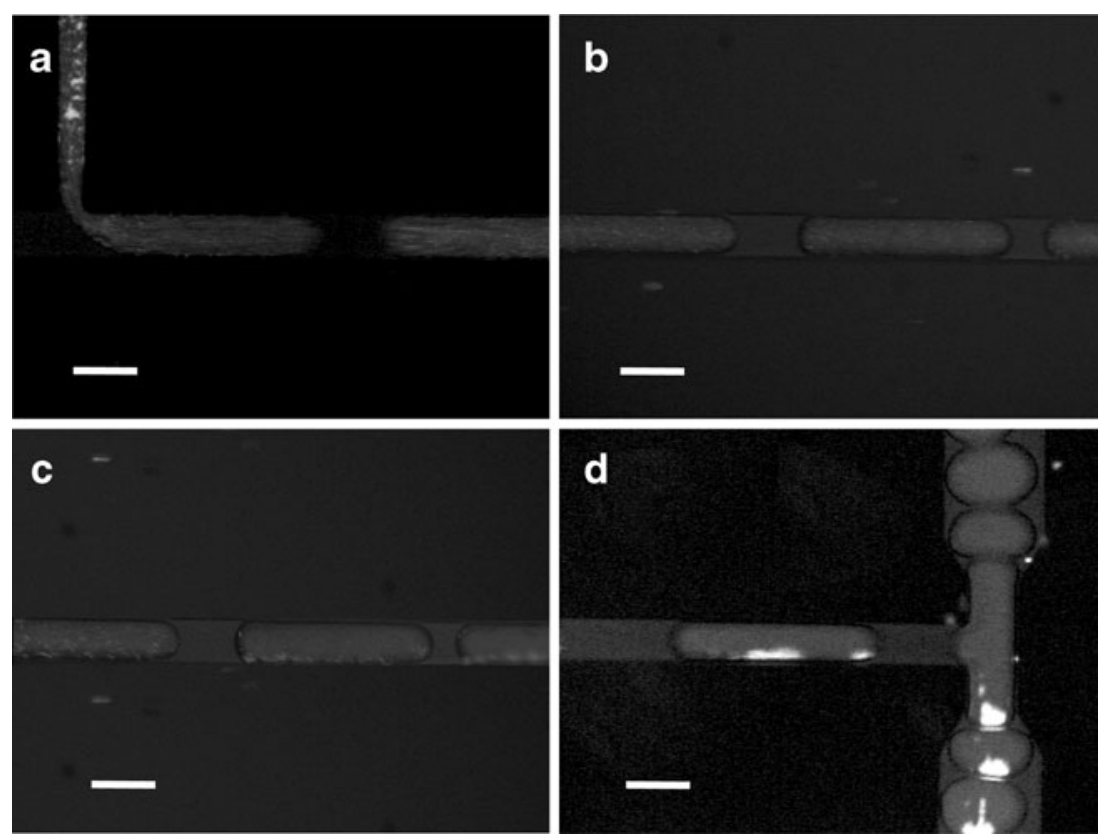

\section{Results and discussion}

Droplets are formed by introducing warfarin and bead suspension into a stream of mineral oil. At this channel section, the beads are not affected by the magnetic field and they usually remain inside the microdroplets. Only occasionally, the loss of a bead into the mineral oil can be observed.

The key feature of the microdevice is the withdrawal of magnetic beads during droplet splitting from one of the daughter droplets. We achieved this by placing a small magnet in close proximity to one of the microchannel outlets. As can be seen in Fig. 2, the beads (here labeled with fluorescein) are already concentrated within one side of the droplet just before splitting. We confirmed the movement of the beads and hence, the mixing inside the droplet, by fluorescence correlation spectroscopy (SI Fig. 1), which is a method that allows determining flow velocities in microfluidic channels [20]. The correlation curves reveal two velocities, the slower velocity corresponds to the speed of the droplets, and the faster velocity can be assigned to the movement of the beads inside the droplets. Once the droplets are exposed to the magnetic field, the autocorrelation function reflects the speed of the droplets only.

Frequently, over $95 \%$ of magnetic beads were collected into the outlet that is close to the magnet (see Fig. $2 \mathrm{~d}$ and Movie S1, Electronic Supplementary Material), for the bead concentration of $3.3 \mathrm{mg} / \mathrm{mL}$ that was used for the experiments (corresponding to $5.6 \times 10^{8}$ beads $/ \mathrm{mL}$ ). Since the droplet volume was around $1.25 \mathrm{~nL}$, each droplet contained about 700 beads. For lower bead concentrations $(<200$ beads per droplet, i.e., $<1 \mathrm{mg} / \mathrm{mL}$ ), we even observed $100 \%$ separation efficiency, i.e., complete withdrawal of beads into one daughter droplet (SI, movie). In general, the method is very robust over a large range of conditions and does not need careful adjustment. A reduced efficiency of the bead separation was only observed for a weak magnetic field (the magnet was placed far away from the microdevice). High flow rates (above $5 \mu \mathrm{L} / \mathrm{min}$ ) and very high bead concentrations $(50 \mathrm{mg} / \mathrm{mL})$ also reduced the performance and in these cases, we observed cross-contamination, i.e., beads were released from a droplet and uptaken by the next droplet. Additional experiments with bigger beads (i.e. $2.8 \mu \mathrm{m}$ diameter instead of $1 \mu \mathrm{m}$ ) were performed, however, the experiments were limited by the settling and adsorption to the bottom of the channel resulting in a reduced amount of the beads inside the droplets (data not shown).

Next, the binding of warfarin to immobilized HSA_MBs was investigated. After successful immobilization of HSA

Table 1 Comparison of measured and reported warfarin-HSA association constant, $K_{\mathrm{A}}$

\begin{tabular}{ll}
\hline Association constant $K_{\mathrm{A}} \times 10^{-5}\left(\mathrm{M}^{-1}\right)$ & Reference \\
\hline $4.74 \pm 0.867( \pm 2.84)^{\mathrm{a}}$ & This work, on-chip method \\
$4.93 \pm 0.371( \pm 2.46)^{\mathrm{a}}$ & This work, bulk method \\
$2.10 \pm 0.2$ & Ref. [37] \\
$6.26 \pm 0.7$ & Ref. [5] \\
\hline
\end{tabular}

\footnotetext{
${ }^{a}$ Mean of three independent experiments \pm standard deviation. The value in brackets includes the error for loading of the beads with HSA. For comparison, the error as calculated in [5] is given as well, i.e., excluding the error for bead-HSA loading
} 
onto the MBs, the beads were incubated with the warfarin solution and an aliquot was injected into the microchip to generate a mono-disperse water-in-oil emulsion. Then, the drops were split into two small volume copies isolating the MBs from free warfarin solution. The warfarin concentrations in the droplets of either side were determined and the association constant can be derived as follows (see also Fig. 1):

$K_{\mathrm{A}}=\frac{\left[\mathrm{W} \_ \text {HSA } \_\mathrm{MBs}\right]}{[\mathrm{W}]_{\text {FREE }}\left[\mathrm{HSA} \_\mathrm{MBs}\right]_{\text {FREE }}}$

where [W_HSA_MBs] is the concentration of warfarin bound to HSA_MBs, $[\mathrm{W}]_{\mathrm{FREE}}$ is the concentration of free warfarin and [HSA_MBs] $]_{\text {FREE }}$ is the concentration of the free binding sites of HSA bound to the MBs, all concentrations for equilibrium conditions. Since the experimental conditions guaranteed that the concentration of the binding sites $\left(3.35 \times 10^{-6} \mathrm{M}\right)$ is much greater than the total warfarin concentration (i.e., around $1 \times 10^{-7} \mathrm{M}$ ) we can assume that $\left[\mathrm{HSA}{ }_{-} \mathrm{MBs}\right]_{\mathrm{FREE}} \approx\left[\mathrm{HSA}{ }_{-} \mathrm{MBs}\right]_{\mathrm{TOTAL}}$.

The concentration of bound warfarin, [W_HSA_MBs], can be derived as follows:

$\left[\mathrm{W} \_\right.$HSA_MBs $]=[\mathrm{W}]_{\text {TOTAL }}-[\mathrm{W}]_{\mathrm{FREE}}$

In our experiments $[\mathrm{W}]_{\mathrm{FREE}}$ and $[\mathrm{W}]_{\mathrm{TOTAL}}$ equal the concentration of warfarin measured after droplet splitting in the droplet fraction without magnetic beads and with magnetic beads, respectively, and are determined by the scintillation counter.

The analysis of the two outlets revealed the concentration ratio of $\left[\mathrm{W} \_\mathrm{HSA} \_\mathrm{MB}\right] /[\mathrm{W}]_{\mathrm{FREE}}=1.59 \pm 0.291$, independent of the initially injected concentrations. Together with the concentration of [HSA_MBs] $]_{\text {TOTAL }}=3.35 \mu \mathrm{M}$ and according Eq. (1), $K_{\mathrm{A}}$ was $(4.74 \pm 2.84) \times 10^{5} \mathrm{M}^{-1}$. It has to be noted that the large standard deviation on the experimental data of $K_{\mathrm{A}}$ is mainly due to the variation of HSA concentration loaded on the beads off-chip (see "Experimental" section), i.e., before introduction into the droplets (without considering this starting error, the standard deviation is $0.867 \mathrm{M}^{-1}$, see Table 1). The method was then benchmarked using a previously known bulk assay (see "Experimental" section). The analysis of the same samples revealed [W_HSA_MB] $/[\mathrm{W}]_{\mathrm{FREE}}=1.66 \pm 0.125$. Thus, $K_{\mathrm{A}}$, was $(4.93 \pm 2.46) \times 10^{5} \mathrm{M}^{-1}$. The results are listed in Table 1 . Our results prove that the microfluidic approach can be reliably employed to determine the warfarin-HSA affinity. While similar investigations of other drugs is straightforward, including competition experiments and displacement studies, an improved detection scheme allowing analysis of individual droplets, e.g., by fluorescence or MS [33-35] and the implementation of electromagnets on the chip [36] will expand the usability of this approach. Further developments that allow the complete separation of the magnetic beads from the droplet (similar to washing steps in a bulk process) would enable to directly discriminate between bound and unbound drug and hence, would expand the possibilities for binding constant determination.

\section{Conclusions}

In summary, the fast and reliable characterization of the warfarin-human serum albumin-binding process was investigated implementing a magnetic beads technique with a microfluidic device for segmented flow. The approach can be regarded as a versatile tool for microfluidic droplet generation, and is most promising in combination with other channel geometries and manipulation modules. The fully continuous method could be employed for various applications, including binding assays, kinetic studies and single cell analysis, in which the fast and complete removal of a reactive component is required. The method can be easily implemented into a modular device with further functionalities as required for pharmacokinetic (ADME) studies. We envision that transfer of drug candidate libraries into microdroplet reactors and the possibility of dropletwise detection will reveal the full potential of the approach for drug discovery and testing studies.

Acknowledgments The authors acknowledge Stefanie Krämer for providing the radio-labeled ${ }^{14} \mathrm{C}$-warfarin and making the scintillation counter available to us. The project was financially supported by the European Research Council under the 7th Framework Program (ERC Starting Grant, project no. 203428, n $\mu$ LIPIDS). We thank Benjamin Cvetkovic and Daniel Schaffhauser for the preparation of the master form and Phillip Kuhn and Simon Küster for the proofreading of the manuscript.

\section{References}

1. He XM, Carter DC (1992) Atomic structure and chemistry of human serum albumin. Nature 358:209-215

2. Dumelin CE, Trussel S, Buller F, Trachsel E, Bootz F, Zhang Y, Mannocci L, Beck SC, Drumea-Mirancea M, Seeliger MW, Baltes C, Muggler T, Kranz F, Rudin M, Melkko S, Scheuermann J, Neri D (2008) A portable albumin binder from a DNA-encoded chemical library. Angew Chem Int Ed 47:3196-3201

3. Lim YT, Lee KY, Lee K, Chung BH (2006) Immobilization of histidine-tagged proteins by magnetic nanoparticles encapsulated with nitrilotriacetic acid (NTA)-phospholipids micelle. Biochem Biophys Res Commun 344:926-930

4. Shukoor MI, Natalio F, Tahir MN, Ksenofontov V (2007) H.A. Therese, P. Theato, H.C. Schroder, W.E. Muller, W. Tremel, Superparamagnetic gamma- $\mathrm{Fe}(2) \mathrm{O}(3)$ nanoparticles with tailored functionality for protein separation. Chem Commun 44:4677-4679

5. Marszall MP, Bucinski A (2010) A protein-coated magnetic beads as a tool for the rapid drug-protein binding study. J Pharm Biomed Anal 52:420-424

6. Srinivasan B, Huang X (2008) Functionalization of magnetic nanoparticles with organic molecules: loading level determination 
and evaluation of linker length effect on immobilization. Chirality 20:265-277

7. Yang C, Guan Y, Xing J, Jia G, Liu H (2006) Synthesis and protein immobilization of monodisperse magnetic spheres with multifunctional groups. React Funct Polym 66:263-273

8. Liu K, Xing J, Guan Y, Shan G, Liu H (2004) Synthesis of aminosilane modified superparamagnetic silica supports and their use for protein immobilization. Colloids Surf A 238:127-131

9. Urban PL, Goodall DM, Bruce NC (2006) Enzymatic microreactors in chemical analysis and kinetic studies. Biotechnol Adv 24:42-57

10. Valera FE, Quaranta M, Moran A, Blacker J, Armstrong A, Cabral JT, Blackmond DG (2010) The flow's the thing... or is it? Assessing the merits of homogeneous reactions in flask and flow. Angew Chem Int Ed 49:2478-2485

11. Dittrich PS, Manz A (2006) Lab-on-a-chip: microfluidics in drug discovery. Nat Rev Drug Discov 5:210-218

12. Utada AS, Lorenceau E, Link DR, Kaplan PD, Stone HA, Weitz DA (2005) Monodisperse double emulsions generated from a microcapillary device. Science 308:537-541

13. Urbanski JP, Thies W, Rhodes C, Amarasinghe S, Thorsen T (2006) Digital microfluidics using soft lithography. Lab Chip 6:96-104

14. Thorsen T, Roberts RW, Arnold FH, Quake SR (2001) Dynamic pattern formation in a vesicle-generating microfluidic device. Phys Rev Lett 86:4163-4166

15. Song H, Ismagilov RF (2003) Millisecond kinetics on a microfluidic chip using nanoliters of reagents. J Am Chem Soc 125:14613-14619

16. Zheng B, Roach LS, Ismagilov RF (2003) Screening of protein crystallization conditions on a microfluidic chip using nanolitersize droplets. J Am Chem Soc 125:11170-11171

17. Chiu DT, Lorenz RM (2009) Chemistry and biology in femtoliter and picoliter volume droplets. Acc Chem Res 42:649-658

18. Li L, Mustafi D, Fu Q, Tereshko V, Chen DL, Tice JD, Ismagilov RF (2006) Nanoliter microfluidic hybrid method for simultaneous screening and optimization validated with crystallization of membrane proteins. Proc Natl Acad Sci 103:19243-19248

19. Song H, Tice JD, Ismagilov RF (2003) A microfluidic system for controlling reaction networks in time. Angew Chem Int Ed 42:768-772

20. Dittrich PS, Jahnz M, Schwille P (2005) A new embedded process for compartmentalized cell-free protein expression and on-line detection in microfluidic devices. Chembiochem 6:811-814

21. Song H, Chen DL, Ismagilov RF (2006) Reactions in droplets in microfluidic channels. Angew Chem Int Ed 45:7336-7356

22. Brouzes E, Medkova M, Savenelli N, Marran D, Twardowski M, Hutchison JB, Rothberg JM, Link DR, Perrimon N, Samuels ML (2009) Droplet microfluidic technology for single-cell highthroughput screening. Proc Natl Acad Sci 106:14195-14200
23. Gijs MA, Lacharme F, Lehmann U (2010) Microfluidic applications of magnetic particles for biological analysis and catalysis. Chem Rev 110:1518-1563

24. Pamme N (2007) Continuous flow separations in microfluidic devices. Lab Chip 7:1644-1659

25. Pamme N (2006) Magnetism and microfluidics. Lab Chip 6:24-38

26. Shah GJ, Kim CJ (2009) Meniscus-assisted high-efficiency magnetic collection and separation for EWOD droplet microfluidics. J Microelectromech Syst 18:363-375

27. Wang Y, Zhao Y, Cho SK (2007) Efficient in-droplet separation of magnetic particles for digital microfluidics. J Micromechanics Microengineering 17:2148-2156

28. Qin D, Xia Y, Whitesides GM (1996) Rapid prototyping of complex structures with feature sizes larger than $20 \mu \mathrm{m}$. Adv Mater 8:3

29. Clausell-Tormos J, Lieber D, Baret JC, El-Harrak A, Miller OJ, Frenz L, Blouwolff J, Humphry KJ, Koster S, Duan H, Holtze C, Weitz DA, Griffiths AD, Merten CA (2008) Droplet-based microfluidic platforms for the encapsulation and screening of Mammalian cells and multicellular organisms. Chem Biol 15:427-437

30. Holtze C, Rowat AC, Agresti JJ, Hutchison JB, Angile FE, Schmitz CH, Koster S, Duan H, Humphry KJ, Scanga RA, Johnson JS, Pisignano D, Weitz DA (2008) Biocompatible surfactants for water-in-fluorocarbon emulsions. Lab Chip 8:1632-1639

31. Moaddel R, Marszall MP, Bighi F, Yang Q, Duan X, Wainer IW (2007) Automated ligand fishing using human serum albumincoated magnetic beads. Anal Chem 79:5414-5417

32. Garstecki P, Fuerstman MJ, Stone HA, Whitesides GM (2006) Formation of droplets and bubbles in a microfluidic T-junctionscaling and mechanism of break-up. Lab Chip 6:437-446

33. Boedicker JQ, Li L, Kline TR, Ismagilov RF (2008) Detecting bacteria and determining their susceptibility to antibiotics by stochastic confinement in nanoliter droplets using plug-based microfluidics. Lab Chip 8:1265-1272

34. Lazar IM, Grym J, Foret F (2006) Microfabricated devices: a new sample introduction approach to mass spectrometry. Mass Spectrom Rev 25:573-594

35. Fidalgo LM, Whyte G, Ruotolo BT, Benesch JL, Stengel F, Abell C, Robinson CV, Huck WT (2009) Coupling microdroplet microreactors with mass spectrometry: reading the contents of single droplets online. Angew Chem Int Ed Engl 48:3665-3668

36. Siegel AC, Shevkoplyas SS, Weibel DB, Bruzewicz DA, Martinez AW, Whitesides GM (2006) Cofabrication of electromagnets and microfluidic systems in poly(dimethylsiloxane). Angew Chem Int Ed Engl 45:6877-6882

37. Loun B, Hage DS (1994) Chiral separation mechanisms in protein-based HPLC columns. 1. Thermodynamic studies of $(R)$ and $(S)$-warfarin binding to immobilized human serum albumin. Anal Chem 66:3814-3822 